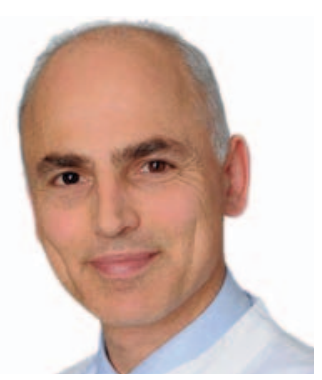

\title{
Eine präoperative Lungenfunktionsprüfung ist unverzichtbar für die Risikoeinschätzung thoraxchirurgischer Patienten
}

\author{
Prof. Dr. Khosro Hekmat
}

Klinik für Herz- und Thoraxchirurgie, Herzzentrum an der Universität zu Köln

\begin{abstract}
Wesolowski SP, Boros PW, Orłowski TM, Quanjer PH: Use the lower limit of normal, not 80\% predicted, in judging eligibility for lung resection. Respiration 2016;92:65-71.
\end{abstract}

Feststellung der Eignung für eine Lungenresektion anhand der Untergrenze des Normbereichs statt $80 \%$ des Sollwerts

\section{Schlüsselwörter}

Lungenfunktionsprüfung · Präoperative Beurteilung ·

Operative Lungenresektion · Lungenkarzinom

\section{Zusammenfassung}

Hintergrund: Eine eingeschränkte Lungenfunktion (LF) ist ein bekannter Risikofaktor für perioperative Komplikationen bei Patienten, bei denen eine operative Lungenresektion angezeigt ist. Die aktuellen europäischen Leitlinien empfehlen, 80\% des Sollwerts als Grenzwert für eine anomale LF heranzuziehen, nicht die Untergrenze des Normbereichs (UN).

Ziele: Ziel war es zu beurteilen, wie sich die Wahl des Grenzwerts (80\% des Sollwerts vs. UN bei -1,645 SA) auf die Inzidenz von Funktionsstörungen und postoperativen Komplikationen bei $\mathrm{Pa}$ tienten mit Lungenkarzinom auswirkt, die zur Lungenresektion überwiesen wurden.
Methoden: Retrospektive Analyse der perioperativen Spirometrie und des Kohlenmonoxid-Transferfaktors $\left(T_{L, C O}\right)$ bei 851 konsekutiven Lungenkarzinompatienten nach operativer Resektion.

Ergebnisse: Bei 369 Patienten (43,4\%) wurde eine Atemwegsobstruktion diagnostiziert, bei 41 Patienten $(4,8 \%)$ ein restriktives Muster. Bei 503 Patienten (59,1\%) lag die exspiratorische Einsekundenkapazität ( $\mathrm{FEV}_{1}$ ) oder der $\mathrm{T}_{\mathrm{L}, \mathrm{CO}}$-Wert unter der UN, bei $620 \mathrm{~Pa}$ tienten $(72,9 \%)$ hingegen betrug einer der beiden Werte $<80 \%$ des Sollwerts (x2-Test: $p<0,0001$ ). Somit hatten 117 von 851 Patienten LF-Werte $<80 \%$ des Sollwerts, aber nicht unter der UN. Die Odds Ratios (OR) für perioperative Komplikationen waren bei den Patienten mit eingeschränkter LF, gemessen an der UN, erhöht $(1,59 ; p=0,0005)$, ausgenommen Patienten mit besonders umfassender Resektion ( $>5$ Segmente). Bei Patienten mit Testergebnissen oberhalb der UN, aber $<80 \%$ des Sollwerts, unterschieden sich die OR für perioperative Komplikationen nicht von denen von Patienten mit normaler LF $(1,14 ; p=0,5)$.

Schlussfolgerungen: Bei Kandidaten für eine Lungenresektion liegt häufig eine Einschränkung der LF vor. Die UN statt 80\% des Sollwerts als Grenzwert zu verwenden, verringert die Prävalenz respiratorischer Beeinträchtigung um 14\% und ermöglicht eine sichere operative Resektion ohne zusätzliche Lungenfunktionstests.

(c) 2017 S. Karger GmbH, Freiburg

\section{KARGER}

Fax +4976145207 14 information@karger.com www.karger.com

(C) 2017 S. Karger GmbH, Freiburg 


\section{Transfer in die Praxis}

\section{Hintergrund}

Die präoperative Risikoabschätzung von Patienten mit Bronchialkarzinom, die sich einem thoraxchirurgischen Eingriff unterziehen sollen, ist sehr komplex. Bedingt durch den Hauptrisikofaktor des Rauchens treten bei sehr vielen Patienten auch eine chronisch obstruktive Lungenerkrankung (COPD) sowie kardiovaskuläre Erkrankungen auf. Die präoperative pneumologische Evaluierung vor einer Lungenresektion kann in eine technische Operabilität sowie eine funktionelle Operabilität eingeteilt werden. Unter der technischen Operabilität versteht man, dass durch die Operation mit einer hohen Wahrscheinlichkeit eine R0-Resektion erreichbar ist. Dies wird im Rahmen eines Tumorboards anhand des CT-Thorax sowie idealerweise eines PET-CTs bestimmt. Da bei jeder anatomischen Lungenresektion auch gesundes Gewebe verloren geht, ist die präoperative lungenfunktionelle Evaluation von großer Bedeutung. Hier empfehlen die Europäischen Gesellschaften für Pneumologie (ERS) und Thoraxchirurgie (ESTS), dass bei der Lungenfunktion die forcierte Einsekundenkapazität $\left(\mathrm{FEV}_{1}\right)$ und die Diffusionskapazität ( $\mathrm{D}_{\mathrm{LCO}}$ ) jeweils mindestens $80 \%$ des Soll betragen sollten [1]. Ist dies der Fall, ist keine weitere Diagnostik notwendig. Sollten die Werte jedoch darunter liegen, empfehlen die ERS und die ESTS die Durchführung einer Spiroergometrie.

\section{Studienergebnisse}

Die vorliegende Arbeit von Wesolowski et al. empfiehlt nunmehr nicht $80 \%$ des Sollwertes als Grenzwert heranzuziehen, sondern stattdessen die Untergröße des Normbereichs zu verwenden. Hierzu wurden retrospektiv 851 konsekutive Lungenkarzinompatienten nach operativer Lungenresektion untersucht. Die Odds Ratios für perioperative Komplikationen waren bei Patienten mit eingeschränkter Lungenfunktion unterhalb der Untergrenze des Normbereichs signifikant erhöht ( $O R=1,59, p=0,0005)$.

Andererseits zeigten Patienten mit Lungenfunktionswerten oberhalb der Untergrenze des Normbereichs, aber $\leq 80 \%$ des Sollwertes, keine Unterschiede bei den perioperativen Komplikationen im Vergleich zu Patienten mit normaler Lungenfunktion. Die Autoren schlussfolgern, dass bei der Verwendung der Untergrenze des Normbereichs einer Lungenfunktion zusätzliche Lungenfunktionstests nicht notwendig sind.

\section{Fazit und Transfer in die Praxis}

Der Vorschlag der Autoren, die Untergrenze des Normbereichs statt $80 \%$ des Sollwertes bei der Bestimmung der FEV 1 sowie der $D_{\mathrm{Lco}} \mathrm{zU}$ verwenden, bringt keinen Vorteil für die Praxis. Die deutsche S3Leitlinie zur Prävention, Diagnostik, Therapie und Nachsorge des Lungenkarzinoms [2] beschreibt bereits andere Cutoff-Werte als die europäische Leitlinie. Hier ist ein Patient bis zu einer Pneumektomie operabel, falls die FEV $1 \geq 2$ I bzw. $\geq 80 \%$ des Soll und die $\mathrm{D}_{\mathrm{LCO}} \geq 60 \%$ des Soll beträgt. Sollten die Werte darunter liegen, wird eine Spiroergometrie empfohlen. Sollte hierbei die maximale Sauerstoffaufnahme über $20 \mathrm{ml} / \mathrm{kg} / \mathrm{min}$ bzw. über $75 \%$ des Soll liegen, ist der Patient ebenfalls bis zu einer Pneumektomie operabel.
In den 1990er Jahren hat das American College of Chest Physicians und die British Thoracic Society festgelegt, dass Patienten mit einer präoperativen $\mathrm{FEV}_{1}$ von mehr als 2 I eine Pneumektomie und jene mit einer präoperativen $\mathrm{FEV}_{1}$ über 1,5 I eine Lobektomie tolerieren. Nach der deutschen S3-Leitlinie kann hier eine Letalitätsrate unter $5 \%$ erwartet werden [2].

Letztendlich muss jedoch bei jedem thoraxchirurgischen Eingriff die Anzahl der zu resezierenden, belüfteten Segmente beachtet werden. Bei einer geplanten Lobektomie sollten nach der Segmentzählmethode die postoperativ berechneten $\mathrm{FEV}_{1}$ - und $\mathrm{D}_{\mathrm{LCO}}$-Werte über 30\% liegen. Zur Quantifizierung von belüfteten und unbelüfteten Lungenarealen hat sich die Lungenperfusionsszintigraphie bewährt.

Außerdem ist auch der Allgemeinzustand des Patienten für die Planung eines operativen Eingriffs von hoher Bedeutung. Der Allgemeinzustand wird in vielen Studien gemäß den Richtlinien der Eastern Cooperative Oncology Group (ECOG) eingeschätzt. Patienten in einem ECOG-Stadium 0-1 können meist problemlos einer operativen Therapie unterzogen werden. Patienten in einem ECOG-Stadium 2 (unfähig zu normaler Aktivität oder Arbeit, selbstversorgend) gelten als Hochrisikopatienten. Patienten im ECOGStadium 3-4 (pflegebedürftig und bettlägerig) sollten keiner operativen Therapie zugeführt werden.

Leider kommt es immer wieder vor, dass Patienten auf Tumorboards mit einer unzureichenden präoperativen Risikoabschätzung vorgestellt werden. Deshalb ist als Mindestmaß für die Vorstellung eines Patienten vor einer geplanten Lungenresektion Folgendes zu fordern:

$1 \mathrm{FEV}_{1}$ in Liter und $\mathrm{D}_{\mathrm{LCO}}$ als Prozentangabe;

2 Spiroergometrie, falls FEV 1 unter 2 I und die DLCo unter 60\%;

3 Einteilung des Allgemeinzustandes nach der ECOG-Klassifikation;

4 Angabe der Nebenerkrankungen mit Hauptaugenmerk auf kardiovaskuläre Erkrankungen;

5 Angabe der Medikation, insbesondere Thrombozytenaggregationshemmer und Antikoagulantien.

\section{Disclosure Statement}

Hiermit erkläre ich, dass keine Interessenskonflikte in Bezug auf den vorliegenden Kommentar bestehen.

\section{Literatur}

1 Brunelli A, Charloux A, Bollinger CT, et al.: ERS/ESTS clinical guidelines on fitness for radical therapy in lung cancer patients (surgery and chemoradiotherapy). Eur Respir J 2009;34:17-41.

2 Goeckenjan G, Sitter H, Thomas M, et al.: Prävention, Diagnostik, Therapie und Nachsorge des Lungenkarzinoms. Pneumologie 2011;65:e51-e75.

Kontaktadresse: Prof. Dr. med. Khosro Hekmat, Klinik für Herz- und Thoraxchirurgie, Herzzentrum an der Universität zu Köln, Kerpener Straße 62, 50931 Köln, Deutschland, khosro.hekmat@uk-koeln.de 